\title{
Der Einfluß hoher Konzentrationen auf die Richtigkeit kinetischer Substrat-Bestimmungen
}

\author{
R. Müller-Matthesius, M. Damm, K. Sommer, Veronika Burk \\ Forschungslaboratorien der Behringwerke AG
}

\section{Zusammenfassung:}

Bei kinetischen Substratbestimmungen muß durch geeignete Meßbedingungen sichergestellt werden, daß oberhalb der Verdünnungsgrenze keine Reagenzerschöpfung eintritt, durch die hohe Konzentrationen zu tief gefunden werden. Diese Fehlerquelle muß z.B. bei kinetischen Harnstoff-Bestimmungen im Hinblick auf die bei Patienten mit extremer Niereninsuffizienz zu erwartenden Höchstkonzentrationen beachtet werden.

\section{Schlüsse/wörter:}

Kinetische Substrat-Bestimmung - fixed-time-Technik - Harnstoff-Bestimmung

\section{Summary:}

For kinetic substrate determination it has to be ensured, that sample concentrations above the measuring range will not cause an early consumption of reagent leading to too low results. This fault has to be excluded e.g. in case of urea determination with respect to the highest concentrations occurring in patients with extreme renal insufficiency.

\section{Keywords:}

Kinetic substrate determination - fixed-time-method - urea determination

\section{Einleitung}

Kinetische Substratbestimmungen weisen bei mechanisierter Durchführung häufig Vorteile gegenüber Endpunkttechniken auf und werden dann bevorzugt angewendet. Ein typisches Beispiel stellt die Bestimmung des Harnstoffs dar. Als sich zeigte, daß das ursprünglich für Endpunktbestimmungen entwickelte vollenzymatische Urease/GIDH-Verfahren (1) eine kinetische Messung erlaubt und damit Vorteile bei der Probendosierung, sowie bei parallel messenden Geräten im Probendurchsatz verbunden sind, wurden die vorhandenen Reaġenziensätze bald für kinetische Harnstoff-Bestimmungen eingesetzt $(2-4)$. Es folgten Optimierungen $(5-8)$ bis hin zu Spezialreagenzien für kinetische Messungen, die als Konsequenz ihrer reduzierten Reaktionsgeschwindigkeit für Endpunktbestimmungen praktisch nicht mehr brauchbar sind. Z.Zt. werden Reagenzien unterschiedlicher chemischer Zusammensetzung kinetisch angewendet.

An Hand einiger exemplarischer Untersuchungen wird eine Fehlermöglichkeit der kinetischen Harnstoff-Bestimmung aufgezeigt, die mit zunehmender Reaktionsgeschwindigkeit der Reagenzien größer wird, durch Wahl geeigneter Meßbedingungen jedoch ausgeschaltet werden kann. Weiterhin haben die Untersuchungen auch deshalb exemplarischen Charakter, weil die gleiche Fehlermöglichkeit bei allen kinetischen Substratbestimmungen, die nach dem fixed-time-Verfahren durchgeführt werden, auszuschließen ist.

Die Untersuchungen wurden deshalb an einem Cobas Bio durchgeführt, weil dieses weitverbreitete Gerät hinsichtlich der Volumen- und Zeiteinstellungen besonders flexibel ist, sich gut für kinetische Substratbestimmungen eignet und hierfür häufig verwendet wird. Grundsätzlich sind die im folgenden beschriebenen Effekte auch bei anderen Meßgeräten zu beachten.

\section{Material und Methoden}

Für die Messungen wurden zwei marktübliche Reagenziensätze aus dem Sortiment der Behringwerke AG eingesetzt: Testomar ${ }^{\circledR}$-Harnstoff Mono $19 \times 15 \mathrm{ml}$ und Testomar-Harnstoff Mono $11 \times 50 \mathrm{ml}$. Das zuerst genannte Reagenz wurde primär für Endpunkt-Bestimmungen entwickelt, reagiert daher relativ schnell und wird im folgenden mit A bezeichnet. Das zweite Reagenz stellt eine Spezialentwicklung für kinetische Messungen dar, reagiert langsamer als $A$ und wird $B$ genannt. Obwohl eine bessere Eignung vollenzymatischer Harnstoff-Reagenzien für kinetische Anwendungen auch durch Zugabe kompetitiver Inhibitoren erreicht werden kann (7), beruht die geringere Reaktionsgeschwindigkeit von Reagenz B nicht auf dem Einsatz solcher Inhibitoren, sondern resultiert aus anderen Konzentrationen bzw. Aktivitäten der beteiligten Reagenzien. Dies ist im vorliegenden Fall deshalb möglich, weil die Enzyme Urease und GIDH von Natur aus hohe Michaelis-Konstanten aufweisen.

Im einzelnen liegen folgende Testkonzentrationen bzw. -aktivitäten vor:

\begin{tabular}{lll}
\hline & Reagenz A & Reagenz B \\
\hline Tris-Puffer & $60 \mathrm{mmol} / \mathrm{I}$ & $60 \mathrm{mmol} / \mathrm{I}$ \\
pH-Wert & 7,5 & 7,8 \\
2-Oxoglutarat & $17 \mathrm{mmol} / \mathrm{I}$ & $8.5 \mathrm{mmol} / \mathrm{I}$ \\
Urease & $4 \mathrm{U} / \mathrm{ml}$ & $7 \mathrm{U} / \mathrm{ml}$ \\
GIDH & $7,5 \mathrm{U} / \mathrm{ml}$ & $3 \mathrm{Ul}$ \\
NADH & $0,23 \mathrm{mmol} / \mathrm{I}$ & $0.22 \mathrm{mmol} / \mathrm{I}$ \\
ADP & $1,3 \mathrm{mmol} / \mathrm{I}$ & - \\
EDTA & $5,7 \mathrm{mmol} / \mathrm{I}$ & - \\
\hline
\end{tabular}

Im Hinblick auf die bei Patienten mit extremer Niereninsuffizienz zu erwartenden Höchstkonzentrationen (400 bis $500 \mathrm{mg} / \mathrm{dl}$ ) wurde eine Harnstoff-Konzentrations- 
reihe zwischen 20 und $1000 \mathrm{mg} / \mathrm{dl}$ durch Einwaage von Harnstoff p.a., Merck, in Wasser hergestellt.

Als Analysengerät wurde der Cobas Bio $₫$ der Firma Hoffmann-La Roche * verwendet. Bei den in Abb. 1 dargestellten Meßwerten wurde das Gerät wie folgt programmiert:

1 Units

2 Calculation factor

$\mathrm{mg} / \mathrm{dl}$

Standard 1 conc

Standard 2 conc

Standard 3 conc

Limit

Temperature ( $\left.{ }^{\circ} \mathrm{C}\right)$

Type of analysis

Wavelength $(\mathrm{nm})$

Sample volume $(\mu l)$

Diluent volume $(\mu \mathrm{l})$

Reagent volume ( $\mu l)$

Incubation time

Start reagent volume

Time of first reading

Time interval

Number of readings

Blanking mode

Print out mode

Diese Einstellungen entsprechen einem bei $25^{\circ} \mathrm{C}$ durchgeführten fixed-time-Verfahren mit $t_{1}=45 \mathrm{sec}$ und $t_{2}$ $=75 \mathrm{sec}$, bei dem alle Konzentrationswerte über einen Standard mit $50 \mathrm{mg} / \mathrm{dl}$ Harnstoff berechnet werden.

- Cobas Bio - eingetragenes Warenzeichen der Fa. Hoffmann-La Roche.

Bei den in Abb. 2 dargestellten Meßwerten wurde bis auf die Temperatureinstellung mit gleicher Programmierung gearbeitet.

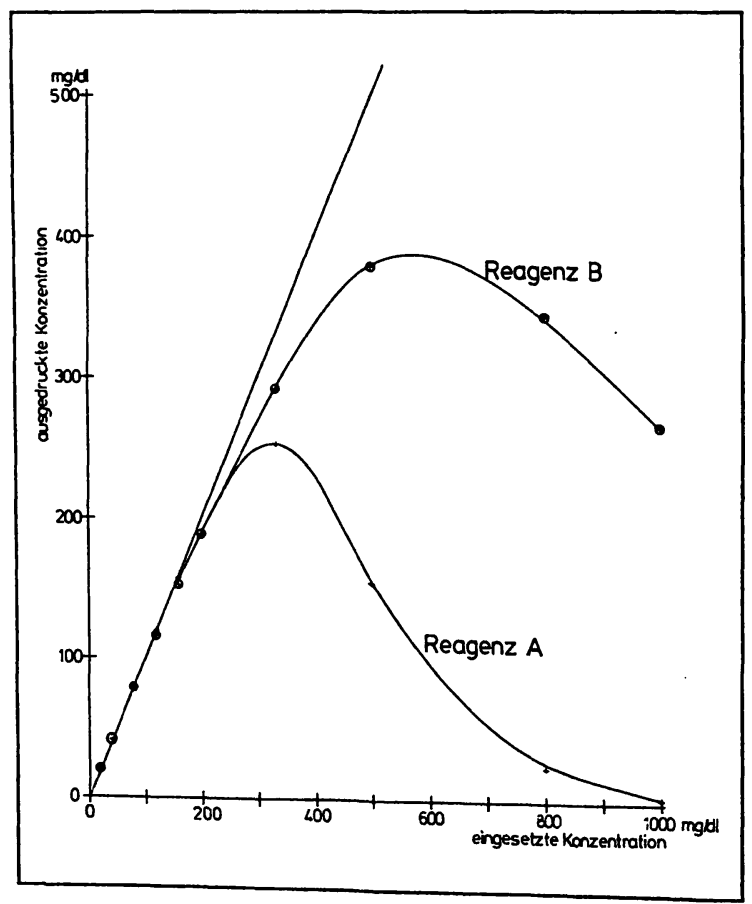

Abb. 1: Wiederfindung der Harnstoff-Konzentration am Cobas Bio unter den im Abschnitt "Material und Methoden" genannten Meßbedingungen. Die Geräteeinstellungen sind im Falle von Reagenz A für Routinemessungen nicht optimal
Die in Abb. 3 dargestellten Extinktions-Zeit-Kurven wurden bei $\mathrm{Hg} 334 \mathrm{~nm}$ und $25^{\circ} \mathrm{C}$ mit einem Eppendorf-Photometer $1101 \mathrm{M}$ mit angeschlossenem Kompensationsschreiber 6511 ermittelt. Ansatz: $1000 \mu$ Reagenz A + $20 \mu l$ Probe.

\section{Ergebnisse und Diskussion}

Bei der Messung wäßriger Harnstoff-Lösungen bis $1000 \mathrm{mg} / \mathrm{dl}$ zeigte sich, daß unter den am Cobas Bio gewählten Einstellungen die ausgedruckten Konzentrationswerte ein Maximum durchlaufen. Dieses lag bei den in Abb. 1 dargestellten Messungen im Falle von Reagenz A bei etwa $300 \mathrm{mg} / \mathrm{dl}$ und im Falle von Reagenz B etwa bei dem doppelten Wert. Aus Abb. 2 geht hervor, daß das Maximum unter geänderten Versuchsbedingungen auch bei anderen Konzentrationen auftreten kann.

Der Wiederabfall der ausgedruckîen Konzentrationen erklärt sich aus einer Reagenzerschöpfung bei hinreichend hohem Harnstoffgehalt der Probe. Aus den in Abb. 3 exemplarisch dargestellten kinetischen Verläufen, die mit Reagenz $A$ unter etwas abweichenden Bedingungen manuell gemessen wurden, ergibt sich im Falle der Konzentration $500 \mathrm{mg} / \mathrm{dl}$ bereits nach einer Minute ein abrupter Stillstand der Extinktion durch Verbrauch des NADH. Dagegen ändert sich im Falle der Lösung mit $40 \mathrm{mg} / \mathrm{dl}$ die Extinktion über einen vergleichsweise längeren Zeitraum. Aus diesem Reaktionsverhalten wird verständlich, daß bei höheren Konzentrationen ein geringeres $\Delta \mathrm{E}$ resultieren kann als bei niedriger Konzentration, wenn die Messung entsprechend spät erfolgt. Betrachtet man beispielsweise das Meßintervall $t_{1}=60 \mathrm{sec}$ und $t_{2}=120 \mathrm{sec}$, so ergibt sich ein $\triangle E$-Wert von etwa 0,33 bei $40 \mathrm{mg} / \mathrm{dl}$ Harnstoff und ein $\triangle \mathrm{E}$-Wert von Null bei $500 \mathrm{mg} / \mathrm{dl}$, weil das NADH bei dieser hohen Konzentration bereits nach $60 \mathrm{sec}$ verbraucht ist.

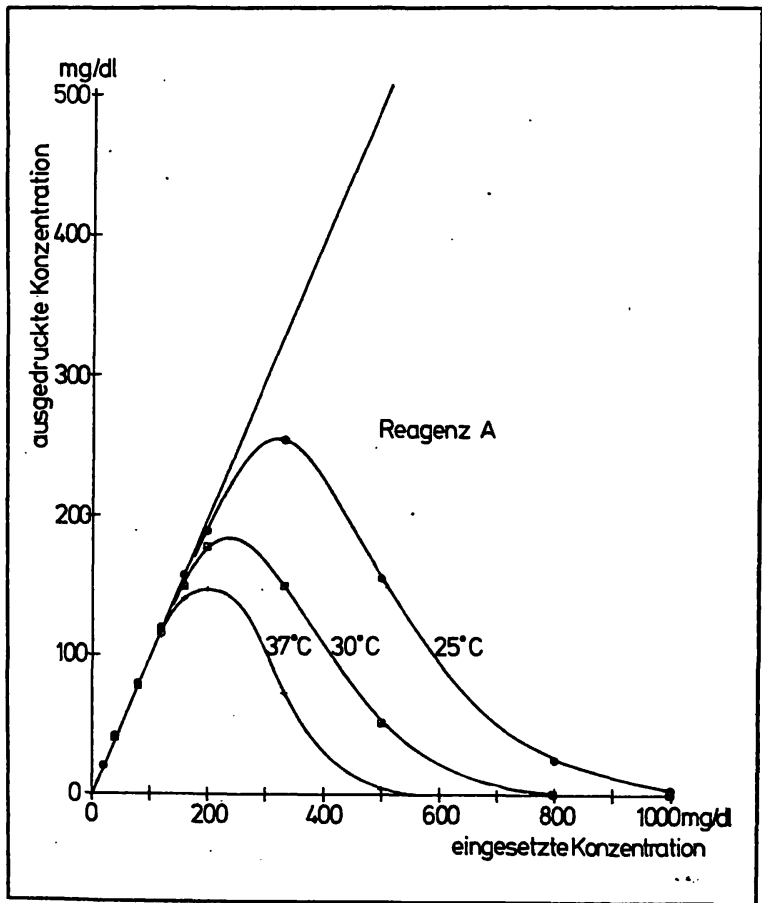

Abb. 2: Abhängigkeit der Standardkurven von der Meßtemperatur. Messungen mit Reagenz A, Geräteeinstellungen siehe "Material und Methoden" 


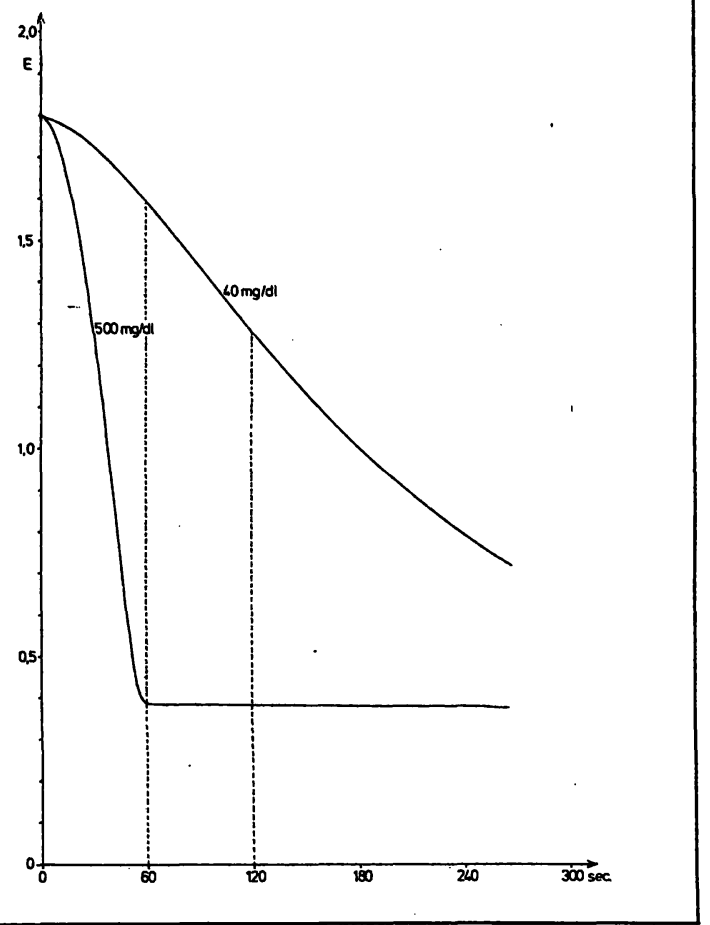

Abb. 3: Zeitliche Extinktionsänderung bei zwei stark unterschiedlichen Harnstoff-Konzentrationen. Manuelle Messung mit Reagenz A

Wie die Lage des $\Delta \mathrm{E}$-Maximums von den eingestellten Meßzeiten abhängig ist, wird aus folgender Betrachtung deutlich. Wenn man in Abb. 3 ein Meßintervall von beispielsweise $30 \mathrm{sec}$ auf der Zeitachse von links nach rechts wandern läßt, ergeben sich zunächst für die Konzentration $500 \mathrm{mg} / \mathrm{dl}$ weitaus größere Extinktionsdifferenzen als für die Lösung mit $40 \mathrm{mg} / \mathrm{dl}$ Harnstoff. Zunehmend verändert sich diese Relation jedoch, bis die $\Delta \mathrm{E}$-Werte schließlich bei $40 \mathrm{mg} / \mathrm{dl}$ größer werden als die $\triangle \mathrm{E}$-Werte bei der Konzentration $500 \mathrm{mg} / \mathrm{dl}$.

Die Lage des $\triangle \mathrm{E}$-Maximums ist nicht nur von den gewählten Meßzeiten, sondern auch von anderen Parametern abhängig.

Aus Abb. 1 ergibt sich der Einfluß der chemischen Eigenschaften der Reagenzien: bei etwa gleichem Gehalt an NADH, dessen Konzentration aus meßtechnischen Gründen nicht beliebig hoch gewählt werden kann, beginnt der $\Delta E$-Wert bei dem schnell reagierenden Reagenz $A$ bei geringeren Harnstoff-Konzentrationen abzufallen als bei dem langsamer reagierenden Reagenz $B$.

Der Einfluß der Meßtemperatur geht aus Abb. 2 hervor. Es resultieren mit steigender Temperatur höhere Reaktionsgeschwindigkeiten, die wiederum zu einer Verschiebung der Maxima zu niedrigeren Konzentrationen führen.

Verständlich ist schließlich auch, daß das Verhältnis von Probe zu Reagenz einen wesentlichen Einfluß haben muß: mit steigendem Probenanteil wird das Maximum zu niedrigen Harnstoff-Konzentrationen verschoben.

Die vorstehend beschriebenen Effekte sind besonders zu beachten, wenn Proben von Patienten mit extremer Niereninsuffizienz oder von urämischen Patienten kinetisch gemessen werden. Die Seren solcher Patienten können vor der Dialyse sehr hohe Harnstoffwerte, 400 bis $500 \mathrm{mg} / \mathrm{dl}$, erreichen. Wie aus den Beispielen in Abb. 1 und 2 hervorgeht, können derart hohe Konzentrationen unter bestimmten Meßbedingungen zu sehr kleinen $\Delta \mathrm{E}$ Werten führen, durch die geringe Harnstoff-Konzentrationen vorgetäuscht werden können. So werden beim Cobas Bio im primären Konzentrationsdruck zwar alle Werte durch „over range” gekennzeichnet, die über der programmierten Verdünnungsgrenze liegen. Es ist jedoch primär nicht unterscheidbar, ob hohe oder tiefe Konzentrationen vorliegen, wenn $\Delta \mathrm{E}-$ Werte unter der Verdünnungsgrenze auftreten. Erst wenn durch einen zusätzlichen Programmabruf (Print out mode 3) die zu den Meßzeiten $t_{1}$ und $t_{2}$ gespeicherten Extinktionen $E_{1}$ und $E_{2}$ ausgedruckt werden, erhält man weiteren Aufschluß. So spricht ein kleiner Wert $E_{1}$ in Verbindung mit einer geringen Extinktionsdifferenz für das Vorliegen einer hohen Harnstoff-Konzentration. Ein quantitatives Orientierungsmerkmal würde man durch das Mitführen einer Harnstoff-Lösung mit einer Konzentration erhalten, die an der programmierten Verdünnungsgrenze liegt.

Zweifellos bedeutet die Durchsicht der Einzelextinktionen wie auch das Mitführen einer zusätzlichen Harnstoff-Lösung einen erhöhten Aufwand, den man vermeiden kann, wenn die Meßbedingungen von vornherein einen so groBen Sicherheitsbereich bieten, daß falsch zu niedrige Harnstoffwerte aus physiologischen Gründen nicht vorkommen können. Dies ist sowohl mit Reagenz $A$ als auch mit Reagenz B erreichbar. Bei der Ausarbeitung solcher Meßbedingungen müssen die physiologisch auftretenden Maximalkonzentrationen unter Berücksichtigung der Reagenzstabilität und eventueller Chargenschwankungen ausgeprüft werden. Man darf sich nicht auf die Ermittlung der Verdünnungsgrenze beschränken. Bei der Auswahl der Meßbedingungen müssen über den $\mathrm{Ge}$ sichtspunkt eines großen Sicherheitsbereiches hinaus selbstverständlich eine Reihe weiterer Kriterien berücksichtigt werden wie etwa eine möglichst weitreichende Linearität der Standardkurve und eine befriedigende Präzision auch bei niedrigen Konzentrationen. Insgesamt bietet das für kinetische Harnstoff-Bestimmungen entwickelte Spezialreagenz B bessere Allround-Eigenschaften für kinetische Anwendungen als das Reagenz $A$, das bei Endpunktbestimmungen das Reagenz der Wahl wäre, bei denen auf Grund der anderen Meßmethodik die hier angesprochene Fehlermöglichkeit a priori nicht auftreten kann. Kurve Reagenz B in Abb. 1 ist ein Beispiel für kinetische fixed-time-Bedingungen, bei denen es unter physiologischen Verhältnissen ebenfalls nicht zu einer Verwechslung zwischen hohen und niedrigen Konzentrationen kommen kann und die auch andere Erwartungen an eine moderne Harnstoff-Bestimmung erfüllen.

Es ist zu beachten, daß die übliche Qualitätskontrolle nur bei Höchstkonzentrationen auftretende Fehler, sei es durch Wahl der falschen Meßbedingungen, sei es durch andere Ursachen wie falsches Auflösen der Reagenzien oder Nichtbeachten der Stabilitätsangaben, nicht anzeigt. Sieht man von derartigen Fehlern ab, stellt die kinetische Harnstoff-Bestimmung unter entsprechend ausgearbeiteten Bedingungen bis zu hohen Konzentrationswerten eine schnelle, sichere und präzise Methode zur Bestimmung des Harnstoffs dar.

Schrifttum s. Seite 24

Anschrift der Verfasser:

Dr. R. Müller-Matthesius

Behringwerke AG

3550 Marburg/Lahn 